\title{
Magnetically driven outflows from Jovian circum-planetary accretion disks
}

\begin{abstract}
Ch. Fendt ${ }^{\star}$
Institut für Physik, Universität Potsdam, Am Neuen Palais 10, 14469 Potsdam, Germany

Astrophysikalisches Institut Potsdam, An der Sternwarte 16, 14482 Potsdam, Germany

Received 1 August 2003 / Accepted 6 October 2003

Abstract. We discuss the possibility to launch an outflow from the close vicinity of a protoplanetary core considering a model scenario where the protoplanet surrounded by a circum-planetary accretion disk is located in a circum-stellar disk. For the circum-planetary disk accretion rate we assume $\dot{M}_{\mathrm{cp}} \simeq 6 \times 10^{-5} M_{\mathrm{J}} \mathrm{yr}^{-1}$ implying peak disk temperatures of about $2000 \mathrm{~K}$. The estimated disk ionization degree and Reynolds number allow for a sufficient coupling between the disk matter and the magnetic field. We find that the surface magnetic field strength of the protoplanet is probably not more than $10 \mathrm{G}$, indicating that the global planetary magnetosphere is dominated by the circum-planetary disk magnetic field of $\$ 50 \mathrm{G}$. The existence of a gap between circum-planetary disk and planet seems to be unlikely. The estimated field strength and mass flow rates allow for asymptotic outflow velocities of $\gtrsim 60 \mathrm{~km} \mathrm{~s}^{-1}$. The overall outflow geometry will be governed by the orbital radius, resembling a hollow tube or cone perpendicular the disk. The length of the outflow built up during one orbital period is about $100 \mathrm{AU}$, depending on the outflow velocity. Outflows from circum-planetary disks may be visible in shock excited emission lines along a tube of diameter of the orbital radius and thickness of about 100 protoplanetary radii. We derive particle densities of $3000 \mathrm{~cm}^{-3}$ in this layer. Energetically, protoplanetary outflows cannot survive the interaction with a protostellar outflow. Due to the efficient angular momentum removal by the outflow, we expect the protoplanetary outflow to influence the early planet angular momentum evolution. If this is true, planets which have produced an outflow in earlier times will rotate slower at later times. The mass evolution of the planet is, however, hardly affected as the outflow mass loss rate will be small compared to the mass accumulated by the protoplanetary core.
\end{abstract}

Key words. ISM: jets and outflows - stars: planetary systems: formation - stars: planetary systems: protoplanetary disks

\section{Introduction}

With the discovery of extrasolar planets during the last decade the scientific interest in planet formation has been increased substantially. In particular, with the help of the computational power existing today, it has become possible to perform numerical simulations of a circumstellar accretion disk containing and building up an orbiting protoplanetary core (e.g. Kley 1999; Bryden et al. 1999; Lubow et al. 1999; Kley et al. 2001; D'Angelo et al. 2002; Tanigawa \& Watanabe 2002; D'Angelo et al. 2003a,b; Bate et al. 2003). Although differing in certain aspects (spatial resolution of the region close to the protoplanet, number of dimensions treated) these simulations have provided us with the same general results. In particular, the simulations show how tidal interaction between the protoplanet and the disk material opens up a gap in the circum-stellar accretion disk along the orbit of the planetary core. Mass accretion in the circum-stellar disk, however, continues across the gap. The circum-stellar disk material entering the Roche lobe of the protoplanet becomes captured and finally accreted by the protoplanetary core. The simulations also demonstrate how the

\footnotetext{
* e-mail: cfendt@aip.de
}

accretion stream initially affected by strong shocks waves eventually builds up a circum-planetary sub-disk in almost Keplerian rotation close to the planet.

On the other hand, in the context of astrophysical jets it is well established that outflow formation is causally connected to the presence of an accretion disk and strong magnetic fields (see Blandford \& Payne 1982; Pudritz \& Norman 1986; Camenzind 1990; Shu et al. 1994). This statement holds for a wide range in outflow energy and spatial scale - for young stellar objects, microquasars and active galactic nuclei. Observations as well as theoretical models strongly suggest that astrophysical sources of outflows are (highly) magnetized. The magnetic field is responsible for acceleration and collimation and for lifting the matter from the disk into the outflow.

In the scenario of planet formation within a circum-stellar accretion disk, the presence of a magnetic field can be expected as well. A central protostellar dipolar magnetic field of $1000 \mathrm{G}$ surface field strength may provide only about $20 \mu \mathrm{G}$ at $5 \mathrm{AU}$ distance. This (weak) field, however, may act as a seed field for dynamo action taking place in the circum-stellar accretion disk, in the protoplanetary core, or in the circum-planetary disk. Most of the present-day Solar system planets carry a substantial 
magnetic field. We expect these fields to be present also during the phase of planet formation.

Considering (i) the numerically established existence of accretion disks around protoplanetary cores as a natural feature during the planet formation phase and (ii) the feasibility of a large-scale magnetic field in the protoplanetary environment, the question arises whether the combination of these two factors may lead to the launch of an outflow similar to the phenomenon observed on much larger spatial and energetic scales. Up to date, the literature on this topic is rather rare. To our knowledge, the possible outflow activity from Jupitersized protoplanets has been investigated only by a single paper (Quillen \& Trilling 1998). Applying stationary models of magnetohydrodynamic outflow formation developed for protostars, these authors came to the conclusion that protoplanetary outflows might indeed exist with velocities $\simeq 20 \mathrm{~km} \mathrm{~s}^{-1}$ and mass loss rates $\simeq 10^{-8}$ Jupiter masses per year.

In the present paper we further investigate the feasibility of launching outflows from accretion disks around protoplanets. The paper is organized as follows. Section 2 summarizes the basic ideas of magnetohydrodynamically driven outflows and their possible application to protoplanets. We then discuss the circum-planetary accretion disk. Section 3.1 is devoted to numerical simulations of circum-planetary disk in the literature. We estimate the temperature (Sect. 3.2) and ionization state (Sect. 3.3) of such disks. The next section considers the magnetic field of protoplanet (Sect. 4.1), and circum-planetary disk (Sect. 4.2), and the expected MHD properties of the outflow (Sects. 4.4 and 4.5). Based on the estimated properties of the system we discuss the parameter space for MHD driven outflows from circum-planetary disks and their observational appearance (Sect. 5). We conclude the paper with our summary (Sect. 6).

We will consider a complex model scenario consisting of a number of constituents. In order to label their parameters, we use the following notation. Parameters of the planetary core have the subscript "p", those of the outflow the subscript "out", those of the circum-planetary disk the subscript "cp", those of the central star the subscript " $\star$ ”. Parameters of the presentday Jupiter are denoted by the subscript "J". Further subscripts will be defined in the text. Variables without subscript denote general quantities.

\section{Magnetohydrodynamic (MHD) formation of outflows}

\subsection{The MHD model of astrophysical outflow formation}

We first outline briefly the essential features of MHD formation of outflows. The generally accepted "standard" model understands the outflow as magnetically driven (Blandford \& Payne 1982; Pudritz \& Norman 1986; Camenzind 1990; Shu et al. 1994). Following current theoretical models the outflow originates in the innermost part of a magnetized "star"-disk system (the "star" can be a young stellar object or a collapsed object). Whether the outflow magnetic field is primarily anchored in the accretion disk or in the surface of the central object, is not yet clear. The fact that outflows are observed also from sources containing a central black hole implies that the key magnetic field component is indeed provided by the surrounding accretion disk. The existence of non-relativistic outflows implies that the relativistic character of the source of an outflow cannot be a major constraint for its launching.

We summarize the MHD formation of outflows process as follows.

- The magnetic field necessary for launching can be generated by dynamo action in the "star"-disk system or advected from the interstellar medium.

- A fraction of the accreting plasma is lifted up to higher altitudes by magnetic forces. The matter couples to the largescale magnetic field, launching a slow wind.

- The matter is then accelerated, first by magneto-centrifugal forces, then, further out, by Lorentz forces. Poynting flux is converted to kinetic energy.

- Inertial forces lead to a "bending" of the poloidal field lines (i.e. the induction of a toroidal magnetic field). The toroidal field tension collimates the outflow into a narrow jet.

- The plasma velocity subsequently exceeds the speed of the MHD waves. The asymptotic, super-magnetosonic flow is ballistic and causally decoupled from outer boundary conditions.

- At the point where the outflow front meets the interstellar medium, a bow shock develops.

It has been discussed in the literature that outflows can only be formed in a geometrical configuration underlying a certain degree of axisymmetry (Fendt \& Zinnecker 1998). In the case of a circumplanetary disk-outflow-system, an axisymmetric alignment is indicated as the strong differential rotation of the circum-planetary disk would control the dynamo mechanism to provide magnetic fields (in the disk or in the planet) aligned with the rotational axis.

Recent numerical simulations confirm this scenario of outflow formation which has been developed over many years mainly by stationary MHD models. Axisymmetric MHD simulations have shown the self-collimating property of MHD flows from rotating disks (Ouyed \& Pudritz 1997) also under the decollimating effect of a turbulent magnetic diffusivity (Fendt \& Cemeljic 2002). A stellar wind dominated disk-outflow does not collimate on the spatial scales considered in the simulations (Fendt \& Elstner 2000). Three-dimensional simulations of collimating disk winds prove that strong non-axisymmetric perturbations at the base of the outflow indeed lead to growing MHD instabilities eventually disrupting the outflow flow (Ouyed et al. 2003).

\subsection{The question of protoplanetary outflows}

In this section we identify the critical points for MHD outflow formation in the context of protoplanetary outflows. First, one has to discuss the existence of a circum-planetary accretion disk and their parameters (accretion rate, temperature and degree of ionization). Circum-planetary accretion disks have not yet been investigated in detail theoretically or numerically. 
Therefore, in our estimates we will apply the following approach. We expect the outflow to be launched in the inner part of the circum-planetary disk. For this inner sub-disk, we assume a mass flow rate as determined by the numerical simulations of the circum-stellar accretion disk, and treat the disk structure assuming a standard, thin Shakura-Sunyaev disk.

In the second step we will derived some estimates on the magnetic field structure close to the protoplanetary core. Two scenarios are plausible - an accretion disk equipartition magnetic field or the field of the protoplanet itself.

In general, the numerical simulations of the accretion stream around the protoplanet do not show any indication for outflow activity around the protoplanet. However, we know that astrophysical outflow are launched magnetically. Therefore, we cannot expect at all to detect such a process in a purely hydrodynamic treatment, which, in addition, does not resolve the region close to the planet. Radiation driven or gas pressure winds can be launched from a very hot accretion disk, however, such a scenario is unlikely for protoplanets where relatively cool disk temperatures are required to allow for solid dust grain condensation (Bryden et al. 1999).

\section{The circum-planetary accretion disk}

\subsection{Numerical evidence for circum-planetary accretion disks}

During the last years, a number of papers have been published considering time-dependent hydrodynamic simulations of a circum-stellar accretion disk which is enclosing an orbiting protoplanetary core (Bryden et al. 1999; Lubow et al. 1999; Nelson et al. 2000; Kley et al. 2001; D’Angelo et al. 2002; Bate et al. 2003). In summary, all these simulations have demonstrated how the tidal interaction between the protoplanet and the viscous circum-stellar accretion disk opens a gap along the orbit. The feasibility of such a scenario has been predicted some decades ago (Lin \& Papaloizou 1986, 1993; Artymowicz \& Lubow 1996). A similar dynamical evolution is known from simulations of circum-binary accretion disks (Artymowicz \& Lubow 1994)

Essentially, accretion of matter within the circum-stellar disk continues across the gap. That part of the material which spirals around the Roche lobe of the planetary core will then enter the Roche lobe and finally be captured by the planetary gravitational potential. The spiraling matter generates a density wave propagating into the circum-stellar disk. The path of the material approaching the Roche lobe follows more and more a circum-planetary orbital motion representing the feature of a circum-planetary accretion disk. Depending on the mass of the planetary core the gas orbiting within the circum-planetary disk reaches different rotational regimes. The simulations show that the orbital velocity profile can deviate from a Keplerian rotation by up to $10 \%$ primarily due to the smoothing of the gravitational potential (D'Angelo et al. 2002). An almost circular orbital symmetry is reached close to the central planet. On the other hand, at smaller radii the deviation from the Keplerian velocity is larger.
The governing parameter for our studies is the accretion rate of the circum-planetary disk. In general, we find a good agreement in the above mentioned papers concerning this value. Kley et al. (2001) give an accretion rate towards the planetary core of

$\dot{M}_{\mathrm{cp}} \simeq 6 \times 10^{-5} M_{\mathrm{J}} \mathrm{yr}^{-1}$

a value which is confirmed also by three-dimensional simulations (D'Angelo et al. 2003a). The exact value depends on certain model assumptions for the circum-stellar disk as the disk mass distributed along the orbit $\left(3.5 \times 10^{-3} M_{\odot}\right.$ between 2 and $13 \mathrm{AU})$, the disk height $(h(r)=0.05 r)$ and the viscosity parameter $\left(\alpha \simeq 4 \times 10^{-3}\right)$. Lubow et al. (1999) give accretion rates typical for their simulations of $\dot{M}_{\mathrm{cp}} \simeq 4.5 \times 10^{-5} M_{\mathrm{J} \mathrm{yr}}{ }^{-1}$ for similar disk parameters as Kley et al. (2001). Bryden et al. (1999) obtain accretion rates between $\dot{M}_{\mathrm{cp}} \simeq 10^{-5} \mathrm{MJ}_{\mathrm{Jr}}{ }^{-1}$ and $\dot{M}_{\mathrm{cp}} \simeq 3 \times 10^{-4} M_{\mathrm{J}} \mathrm{yr}^{-1}$ which are, as expected, larger in the case of a higher disk viscosity. In the present paper, we will in general refer to the model parameters of Kley et al. (2001) and D'Angelo et al. (2002) and apply their numerically derived accretion rate of $6 \times 10^{-5} M_{\mathrm{J}} \mathrm{yr}^{-1}$. This value is substantially higher than the value estimated by Quillen \& Trilling (1998).

A difficulty with applying these numerical simulations to the circum-planetary accretion flow is their lack of sufficient numerical resolution in this region. In particular, this holds for the innermost part of the circum-planetary disk where we expect the outflow to be launched. The interesting length scales here are the planetary gravitational radius $r_{\mathrm{H}}=D_{\mathrm{p}}\left(M_{\mathrm{p}} / 3 M_{\star}\right)^{1 / 3}$ (Hill radius),

$r_{\mathrm{H}}=508 R_{\mathrm{J}}\left(\frac{D_{\mathrm{p}}}{5.2 \mathrm{AU}}\right)\left(\frac{M_{\mathrm{p}}}{M_{J}}\right)^{1 / 3}\left(\frac{M_{\star}}{M_{\odot}}\right)^{-1 / 3}$,

the radius of the planetary core of presumably several $R_{\mathrm{J}}$, and the orbital radius $D_{\mathrm{p}}$.

Recent simulations by Tanigawa \& Watanabe (2002), particularly considering the gas accretion flow close to the protoplanet, apply a numerical grid extending from $0.025 r_{\mathrm{H}}$ to $12 r_{\mathrm{H}}$ resolving $0.005 r_{\mathrm{H}}$ close to the planet. For parameters similar to Eq. (2) this corresponds to about $\simeq 20 R_{\mathrm{J}}$ and covers reasonably well the region where we expect outflow formation to happen. Due to a different setup - no gap in the circum-stellar disk along the orbit - the accretion rate towards the planetary core is higher by factor of hundred compared to the literature (Lubow et al. 1999; D’Angelo et al. 2002). Compensating for this effect the authors confirm values for the re-scaled surface densities and accretion rates similar to Eq. (1). Further, Tanigawa \& Watanabe deal with an isothermal disk, and, thus, cannot deliver the temperature profile in the disk. The authors claim that the main features of the flow dynamics are roughly the same for adiabatic and isothermal simulations (with a factor 3 difference in the accretion rate).

Due to lack of numerical resolution close to the planet, the simulations published so far give little information about the structure of the circum-planetary disk (e.g. Kley 2001). Threedimensional simulations are essential to determine the scale height $(h / r)$ of the sub-disk. Further, the disk structure also depends on the number value for the viscosity parameter which is 
generally taken constant throughout the whole computational domain (in particular, it is assumed to be the same for circumstellar and circum-planetary disk). So far, the simulations have shown that the scale height of the sub-disk is much smaller compared to the main disk due to the gravitational potential of the planet (Bate et al. 2003).

Preliminary results of very high resolution numerical simulations (Ciecielag et al. 2000) suggest that the spiral density wave launched in the outer regions of the circum-planetary disk may propagate into the inner disk regions. However, simulations by Bate et al. (2003) indicate that the strong spiral shocks observed in 2D simulations are greatly diminished in 3D simulations. In recent simulations by D'Angelo (2003) the circumplanetary disk becomes almost axisymmetric in the region within $20 R_{\mathrm{J}}$ from the planet. In the end, it is up to future highresolution circum-planetary disk simulations to show whether the simple assumption of an axisymmetric, thin Keplerian disk must be revised.

In summary, numerical hydrodynamic simulations of circum-stellar accretion disks give clear evidence for the existence of circum-planetary disks in almost Keplerian rotation. In order to understand further the accretion process towards the protoplanetary core, the numerical simulation of the circumplanetary accretion disk itself is essential. No such work has yet been presented in the literature. Therefore, we have to rely on estimates obtained by scaling the standard accretion disk parameters to protoplanetary parameters taking into account the results of the above mentioned circum-stellar disk simulations as model constraints. In the following we will consider a thin disk with accretion rate as given in Eq. (1).

\subsection{Circum-planetary disk temperature and density}

Only matter of a sufficiently hot and, thus, sufficiently ionized accretion disk can couple to and interact with a magnetic field, eventually leading to the launch of winds or outflows. Therefore, we first need an estimate for the disk temperature profile. As discussed above, in order to estimate the temperature profile of the circum-planetary accretion disk, we will consider the limit of a standard (thin, Shakura-Sunyaev) disk (see also Nelson \& Benz 2003) with an accretion rate Eq. (1) provided by the numerical circum-stellar disk simulations.

A first estimate of the circum-planetary disk temperature can be derived from the well known equation for the effective surface temperature of a geometrically thin disk surrounding a central body of mass $M$ and an accretion rate $\dot{M}$ as a function of radius $r$ is

$T_{\mathrm{s}}(r)=\left(\frac{3}{8 \pi \sigma} \frac{G M \dot{M}}{r^{3}}\right)^{1 / 4}$

with the Stefan-Boltzmann constant $\sigma$ (see e.g. Pringle 1981). For Jupiter-like parameters and a circum-planetary disk accretion rate as in Eq. (1) we obtain

$T_{\mathrm{s}}(r)=1280 \mathrm{~K}\left(\frac{M}{M_{\mathrm{J}}}\right)^{1 / 4}\left(\frac{\dot{M}_{\mathrm{cp}}}{6 \times 10^{-5} M_{\mathrm{J} \mathrm{yr}^{-1}}}\right)^{1 / 4}\left(\frac{r}{10 R_{\mathrm{J}}}\right)^{-3 / 4}$

Interestingly, we see that the "power" of the disk $\sim M \dot{M}$ normalized to the mass of the central object is similar for the circum-planetary and the circum-stellar case. However, the circum-planetary disk extends a factor $\left(R_{\odot} / R_{\mathrm{J}}\right)$ closer to the origin of the gravitational potential. Therefore, a high temperature and luminosity can be expected in the inner disk.

The disk temperature as estimated in Eq. (3) is substantially larger than that of Quillen \& Trilling (1998). This is due to the much higher accretion rate we consider as motivated by the numerical simulations. The temperature derived in Eq. (3) is a measure for the disk surface temperature.

Viscous heating of the disk will lead to even higher temperatures. For comparison, we show the mid-plane temperature distribution in the case of the $\alpha$-parametrization for the viscosity (Shakura \& Sunyaev 1973) in the appendix. As a result, we find a remarkable similarity between the circum-planetary and the circum-stellar accretion disks.

A straightforward estimate of the mid-plane disk temperature can be made by assuming radiative diffusion in the disk. Then the effective black body surface temperature is related to the central mid-plane temperature by $T_{\mathrm{c}}^{4}=(3 / 8) \tau T_{\mathrm{eff}}^{4}$ (Hubeny 1990). This gives mid-plane temperatures $\simeq 2-4$ higher than the surface temperature and rather insensitive to the vertical optical depth $\tau$. Our simple estimates yielding a rather high circumplanetary accretion disk temperature is in agreement also with disk models discussing the formation of the Galilean satellites (Canup \& Ward 2002).

In order to derive a realistic disk internal temperature profile, more sophisticated theoretical model calculations are needed taking into account radiation transfer and a realistic description of the opacity. Only few such models are available for protostellar accretion disks (e.g. D’Alessio et al. 1998; Malbet et al. 2001; Dullemond et al. 2002), none for circum-planetary disks. For the example of T Tauri star accretion disks, it has been found (Malbet et al. 2001) that the central disk temperature is a factor ten higher than the surface temperature within a disk radius of about $10 \mathrm{AU}$. Malbet et al. have pointed out that the other important heating source for the disk besides viscosity, namely the radiation of the central $4000 \mathrm{~K}$ star has not been taking into account in their model. D'Alessio et al. (1998) consider stellar irradiation as the main heating source of the disk upper layers. Similarly, Tanigawa \& Watanabe (2002) have argued that the temperature profile of a circum-planetary subdisk within the Hill radius is substantially affected by heating of the protoplanetary core, in particular in the late stages of planetary growth. For our estimates, we do not consider the detailed vertical structure of the disk and the effect of irradiation. Our simple temperature estimate must be therefore considered as a lower limit.

Recent 2D thermo-hydrodynamic simulations (D'Angelo et al. 2003b) were able to resolve the temperature profile close to the planet with reasonable resolution. These simulations take into account radiative heating and cooling as well as a sophisticated opacity model. The authors find a peak mid-plane temperature of $1200-1600 \mathrm{~K}$ at radii of $r=5-10 R_{\mathrm{J}}$ from the protoplanet (D'Angelo 2003). Essentially, these temperatures are somewhat below the values given by our simple ansatz of a standard disk. This agrees with models of circum-stellar disks taking into account radiative transfer and which give in 
general a lower mid-plane temperatures compared to the treatment without radiative transfer (Dullemond et al. 2002).

A very high disk temperature might be problematic as potentially leading to a disk evaporation as discussed for disks around collapsed objects (e.g. Meyer \& Meyer-Hofmeister 1994). Disk evaporation would affect just that (innermost) part of the circum-planetary disk where we expect the origin of the outflow. However, due to the fact that the circum-planetary disk reaches deeper into the gravitational potential of the central body, its $\alpha$-disk scale height is less compared to the case of a circum-stellar disk (see Eq. (A.5)). This, and also the general similarity to the circum-stellar disk, indicates that the circumplanetary disk does not blow up and remains a thin disk close to the planet.

\subsection{The circum-planetary disk ionization}

A minimum degree of ionization is necessary to couple the matter to the magnetic field sufficiently. In the case of protostellar accretion disks several sources of disk ionization have been identified, (i) thermal ionization, (ii) ionization by cosmic ray particles, and (iii) ionization by non-thermal X-rays. As discussed above, the temperature and density profiles are comparable in circum-stellar and circum-planetary accretion disks. Since the circum-planetary disk is located within the circumstellar disk, it is straight forward to assume that also the ionization sources are the same, indicating that also the the degree of disk ionization will be similar in both cases.

To determine the ionization degree of a protostellar accretion disk is a complex enterprise and depends on a number of unknown quantities (see e.g. Gammie 1996; D'Alessio et al. 1998; Fromang et al. 2002). In our case, we are interested in the innermost part of the circum-planetary accretion disk, where the launch of an outflow may take place. In this region the disk is hot and dominated by thermal ionization. The degree of ionization is then

$$
\begin{aligned}
\xi= & 4.2 \times 10^{-13}\left(\frac{a}{10^{-7}}\right)^{1 / 2}\left(\frac{T_{\mathrm{c}}}{10^{3} \mathrm{~K}}\right)^{3 / 4} \\
& \times\left(\frac{n_{\mathrm{n}}}{10^{15} \mathrm{~cm}^{-3}}\right)^{-1 / 2} \frac{\exp \left(-25188 T^{-1}\right)}{1.15 \times 10^{-11}}
\end{aligned}
$$

with the neutral particle density $n_{\mathrm{n}}$ and $a$ the abundance of $\mathrm{K}$ atoms relative to hydrogen (see Fromang et al. 2002 and references therein). Thermal ionization is dominant for midplane temperatures $\gtrsim 10^{3} \mathrm{~K}$. Applying our previous estimates for the temperature profile in circum-planetary disks, such temperatures can be expected for radii $r \lessgtr 65 R_{\mathrm{J}}$. Considering density values as given in Eq. (A.1) we find an ionization degree above $10^{-11}$ for $r \lesssim 65 R_{\mathrm{J}}$.

Note that the degree of thermal ionization of the circumstellar disk at the orbital radius of 5.2 AU is much lower (Eq. (4)) and negligible. However, cosmic ray ionization may still provide ionization levels up to $10^{-11}$ at these radii (Gammie $^{1}$ 1996, see Eq. (5)) and may therefore also contribute to the circum-planetary disk ionization. The vertical

\footnotetext{
${ }^{1}$ There are still large uncertainties in the ionization rate along the disk surface, mostly connected to the possible impact of the stellar wind.
}

profile of the ionization level can only be calculated numerically. Model simulations of circum-stellar disks indicate that lower disk layers may actually be decoupled from the magnetic field (Fromang et al. 2002). This has already been discussed by Gammie (1996) who pointed out sufficient coupling between matter and field is essential in order to excite the disk turbulence via the magneto-rotational instability (Balbus \& Hawley 1991). The results by Gammie further indicate a two-layered accretion structure in protostellar accretion disks for radii $>0.1 \mathrm{AU}$. Regions in which the magnetic Reynolds number is $R e_{\mathrm{M}}<1$ are magnetically "inactive".

It is interesting to work out these numbers for a circumplanetary disk. The magnetic Reynolds number is related to a certain degree of ionization. For the case of a circum-planetary disk, we obtain

$R e_{\mathrm{M}}=7.6 \times 10^{15} \xi \alpha^{1 / 2}\left(\frac{r}{R_{\mathrm{J}}}\right)^{3 / 2}\left(\frac{T}{500 \mathrm{~K}}\right)\left(\frac{M}{M_{\mathrm{J}}}\right)^{-1 / 2}$

Using the number values derived above, we see that in the inner part of the disk a sufficient ionization level $\xi \gtrsim 10^{-15}$ is always guaranteed providing a good coupling of matter and magnetic field as an essential condition in order to launch an outflow.

\section{The magnetic environment of the protoplanet}

We know from other astrophysical sources that outflow formation is basically a magnetic phenomenon - outflows are launched, accelerated and collimated by magnetic forces. Similar to the case of protostars and protostellar outflows, two model scenarios for the magnetic field structure around the protoplanetary core seem to be feasible. One is that of a magnetic field distribution dominated by the protoplanetary magnetosphere. In the other, the accretion disk magnetic field is the main driver for the outflow. In the following we will elaborate in greater detail the scenario of a magnetohydrodynamic interrelation between the protoplanetary core, surrounding accretion disk and a possible outflow.

\subsection{The protoplanetary magnetosphere}

Many of the solar system planets carry a substantial magnetic field, in particular the outer gas giants. Jupiter has a well ordered, strong dipolar magnetic field of about $10 \mathrm{G}$ aligned with the rotational axis. It must be mentioned, however, that the detailed structure and orientation of the solar system planets differs substantially and the theoretical understanding of the underlying (necessarily diverse) dynamo processes is not yet understood (e.g. Connerney 1993; Moss \& Brandenburg 1995).

So far, we are not aware of any theoretical treatment about the magnetic field evolution in protoplanets. Therefore, our considerations of the structure and field strength of the protoplanetary magnetosphere have to rely on simplifying estimates.

Nevertheless, we know from the comparison of stars and protostars that the large scale magnetic field strength observed in young stars is generally higher than the field strength found in main sequence stars. The main reason is that the strength of stellar dynamo action depends on convection (turbulence) 
and rotation (see Bouvier 1990; Ghosh 1995 and references therein). These parameters are clearly more pronounced in the early phase of star formation. It might be straight forward to assume a similar extrapolation for the early formation phase of a planet. This would imply that the large-scale protoplanetary magnetic field has been larger than that of a present-day planet. The seed magnetic field for the protoplanetary dynamo can be provided by the magnetosphere of the central star and/or the circum-stellar accretion disk where the planet is embedded in. What kind of dynamo ( $\alpha^{2}$ or $\alpha \Omega$ ) is operating in a protoplanet is not known. Similar to protostars a dynamo driven by turbulent convection $(\alpha)$ is therefore feasible. Whether, in addition, differential rotation $(\Omega)$ plays a role remains unclear. It has been shown that fully convective protostars do not rotate differentially (see below). There is theoretical indication from the planet formation that a giant gaseous protoplanet reaches an almost fully convective state within some hundred years (Wuchterl et al. 2000). However, as for protostars, the presence of an accretion disk and the inferred magnetic coupling between disk and planet will supply a substantial amount of differential rotation. Thus, both kinds of dynamo action can be expected, an $\alpha \Omega$-dynamo producing an aligned axisymmetric dipole, or an $\alpha^{2}$-dynamo with a non-axisymmetric field structure. Also a quadrupolar field structure are feasible, in particular for high Taylor numbers (Grote et al. 1999).

The maximum field strength which could be generated by a dynamo can be estimated considering the fact that convection must be super-Alfvénic (Camenzind 1997; see also Küker \& Rüdiger 1999). The Alfvén speed for this critical (mean) field strength is $v_{\mathrm{A}} \simeq B_{\mathrm{cr}} / \sqrt{4 \pi \bar{\rho}}$ and has to be compared to the convective velocity defined by the convective turnover time scale $\tau_{\text {con }}$. The convective velocity $v_{\mathrm{c}}$ can be derived from the energy transport in the protoplanet. Considering the convective radiation flux in the limit of mixing length theory, the luminosity of the protoplanet is $L_{\mathrm{p}}=\epsilon_{\mathrm{c}} M_{\mathrm{p}} v_{\mathrm{c}}^{3} / R_{\mathrm{P}}$.

Theoretical models of the planetary evolution have shown that the luminosity of a young planet can be very high, $L_{\mathrm{p}} \simeq$ $10^{-4} L_{\odot}$ (Burrows et al. 2001). Therefore, we estimate the mean convective velocity in a protoplanet as

$v_{\mathrm{c}}=14 \mathrm{~m} \mathrm{~s}^{-1}\left(\frac{\epsilon_{\mathrm{c}}}{30}\right)^{-1 / 3}\left(\frac{L_{\mathrm{p}}}{10^{-4} L_{\odot}}\right)^{1 / 3}\left(\frac{M_{\mathrm{p}}}{M_{\mathrm{J}}}\right)^{-1 / 3}\left(\frac{R_{\mathrm{P}}}{3 R_{\mathrm{J}}}\right)^{1 / 3}$.

Comparison to the mean Alfvén speed in the planet provides the critical magnetic field strength for a hypothetical protoplanetary dynamo,

$$
B_{\mathrm{cr}}=10 \mathrm{G}\left(\frac{\epsilon_{\mathrm{c}}}{30}\right)^{-1 / 3}\left(\frac{M_{\mathrm{T}}}{0.2}\right)^{-1}\left(\frac{L_{\mathrm{p}}}{10^{-4} L_{\odot}}\right)^{1 / 3}\left(\frac{M_{\mathrm{p}}}{M_{\mathrm{J}}}\right)^{1 / 6}\left(\frac{R_{\mathrm{P}}}{3 R_{\mathrm{J}}}\right)^{-7 / 6}
$$

with the Alfvén Mach number of the convective motion $M_{\mathrm{T}} \equiv$ $v_{\text {con }} / v_{\mathrm{A}}$. A higher field strength would imply a magnetic quenching mechanism suppressing the convective motion (and the turbulence) which drives the dynamo.

This estimate of an upper limit for the magnetic field strength in fully convective protoplanets is interesting in two respects. Firstly, the derived field strength is not higher than the field strength observed in present-day solar system planets. If this similarity is true, it indicates a substantial difference between the magnetic evolution of protostars and protoplanets. Secondly, this field strength is well below the equipartition field strength in the circum-planetary accretion disk (see below). Therefore, we believe that, in difference to the case of protostars, the protoplanetary magnetic field cannot dominate the dynamics of the system even close to the planetary core.

It has been found that a good indicator for the level of magnetic activity of a star with rotational period $P$ is the Rossby number $R o=P / \tau_{\text {con }}$ (see Pizzolato et al. 2003). If we adapt this relation for the case of a protoplanet we find

$$
\begin{aligned}
R o= & 2.3 \times 10^{-3}\left(\frac{\Omega_{\mathrm{p}}}{\Omega_{K}\left(R_{\mathrm{P}}\right)}\right)^{-1}\left(\frac{\epsilon_{\mathrm{con}}}{30}\right)^{1 / 3}\left(\frac{L_{\mathrm{p}}}{10^{-4} L_{\odot}}\right)^{1 / 3} \\
& \times\left(\frac{M_{\mathrm{p}}}{10^{-3} M_{\odot}}\right)^{-5 / 6}\left(\frac{R_{\mathrm{P}}}{3 R_{\mathrm{J}}}\right)^{5 / 6} \cdot
\end{aligned}
$$

Compared to protostars (Camenzind 1997), this value is somewhat higher (factor 3/2), indicating that the protoplanet can exhibit a high magnetic activity as the central protostar. However, the high level of activity is probably compensated by the weaker energy output due to the relatively low surface magnetic field strength.

So far we have neglected the effect of dissipative processes for the dynamo mechanism. Diffusive and viscous effects will certainly determine the dynamical evolution of the system. These effects are widely unstudied, and probably not yet fully understood (see e.g. Blackman 2003; Vishniac et al. 2003). Therefore, in the following we will discuss some general characteristics of the protostellar dynamo by estimating dimensionless dynamo numbers (see Camenzind 1997; Küker \& Rüdiger 1999) in the framework of the mean field dynamo theory.

The first point to note is that the low Rossby number $R o \ll$ 1 indicates the protoplanet as a fast rotator. Thus, the scale of the dynamo $\alpha$-effect, the $\alpha$-dynamo parameter $\alpha_{\mathrm{D}}$, is given by the convective velocity $\alpha_{\mathrm{D}} \simeq v_{\mathrm{c}}$. Another interesting question is whether the $\alpha$ or $\Omega$-effect plays the major role for the dynamo. This can be estimated by comparing the magnetic Reynolds numbers $R_{\alpha} \equiv \alpha_{\mathrm{D}} R_{\mathrm{P}} / \eta$ and $R_{\Omega} \equiv \Delta \Omega_{\mathrm{P}} R_{\mathrm{P}}^{2} / \eta$, where $\eta$ is the turbulent magnetic diffusivity in the planet and $\Delta \Omega_{\mathrm{P}}$ the differential rotation. As a matter of fact, the latter quantity is quite unknown. For $\Delta \Omega_{\mathrm{P}} \simeq \Omega_{\mathrm{P}}$ we find a maximum ratio $\left(R_{\Omega} / R_{\alpha}\right) \simeq$ $\Omega_{\mathrm{P}} R_{\mathrm{P}} / v_{\mathrm{c}} \simeq 10^{2}$. On the other hand, it has been shown that fully convective protostars rotate almost rigidly (Küker \& Rüdiger 1997). This holds in general for spherical stars without latitudinal gradients in pressure, density and temperature. We are attempted to extrapolate these results to fully convective protoplanetary cores. As a consequence, $\Delta \Omega_{\mathrm{P}} \ll \Omega_{\mathrm{P}}$ and the mean field dynamo is dominated by the alpha-effect (turbulence).

The $\alpha$-effect becomes more efficient for faster rotators, as noted by Küker \& Rüdiger (1999) for protostars. Also this statement can certainly be generalized from protostars to protoplanets. In difference to protostars, the protoplanetary core is colder. Thus, the magnetic Prandtl number (measuring viscosity in terms of diffusivity) is generally small. For planets we have only $P_{\mathrm{m}}=(v / \eta) \sim 10^{-5} \ldots 10^{-2}$ (see also Kirk \& Stevenson 1987; Starchencko \& Jones 2002). In turn, this implies that the time scale for the turbulent planetary dynamo is very short. We expect a rapid increase in field strength during 
the linear regime up to a substantial value, and later a slow saturation to the maximum field strength (non-linear quenching of the dynamo-driving turbulence).

\subsection{Magnetosphere-disk interaction}

We now address the question whether the planetary magnetic field may, similar to the case of protostars, disrupt the inner part of the disk forming a gap between the planet and an inner disk radius. The gas pressure $p$ in a standard disk with an accretion rate $\dot{M}$ and a disk half thickness $h(r)$ around a central body of mass $M$ is (neglecting factors of orders of unity)

$p(r)=\frac{1}{\alpha} \frac{\dot{M}}{4 \pi r^{2}}\left(\frac{G M}{r}\right)^{1 / 2}\left(\frac{h}{r}\right)^{-1}$

(Camenzind 1990; see also Rüdiger et al. 1995). The quantity $\alpha$ is the usual disk viscosity parameter for the viscous shear stress $t_{r \phi}=-\alpha p$. For a dipolar magnetic field with $B \sim r^{-3}$, pressure equilibrium between the magnetic pressure of the protoplanetary magnetosphere and the accretion disk gas pressure defines the inner disk radius $r_{\text {in }}$. In units of the radius of the central object $R$, the inner disk radius is located at

$\frac{r_{\text {in }}}{R}=\left(\frac{\alpha}{2}\right)^{2 / 7}\left(\frac{G M}{R}\right)^{-1 / 7}\left(\frac{h}{r}\right)^{2 / 7}\left(B_{\mathrm{s}} R\right)^{4 / 7} \dot{M}^{-2 / 7}$.

Here, $B_{\mathrm{S}}$ is the magnetic field strength on the surface of the central body. If the magnetic pressure is strong enough, it will open up a gap between the surface of the central body and the disk. In the case of protostellar magnetospheres, the inner disk radius is located at about 3 protostellar radii (Camenzind 1990; Königl 1991). However, for the case of a circum-planetary accretion disk and a $10 \mathrm{G}$ central dipolar planetary magnetic field the situation would be quite different,

$$
\begin{aligned}
\frac{r_{\mathrm{in}}}{R_{\mathrm{P}}}= & 0.11 \alpha^{2 / 7}\left(\frac{B_{\mathrm{S}}}{10 \mathrm{G}}\right)^{4 / 7}\left(\frac{\dot{M}_{\mathrm{cp}}}{6 \times 10^{-5} M_{\mathrm{J} \mathrm{yr}^{-1}}}\right)^{-2 / 7} \\
& \times\left(\frac{M}{M_{\mathrm{J}}}\right)^{-1 / 7}\left(\frac{R_{\mathrm{P}}}{3 R_{\mathrm{J}}}\right)^{5 / 7}\left(\frac{h / r}{0.1}\right)^{2 / 7} \cdot
\end{aligned}
$$

Such a low (academic) value for the inner disk radius indicates that for the accretion rate considered here the dynamical impact of the planetary magnetosphere for the disk structure is small. Only for a $50 \mathrm{G}$ surface field strength in combination with an accretion rate 100 times lower the inner disk radius moves away from the surface of the planet to a radius $r_{\text {in }} \simeq 2 R_{\mathrm{P}}$. This estimate is consistent with the results of Quillen \& Trilling (1998) who derive $r_{\text {in }} \simeq 1.3 R_{\mathrm{P}}$. Quillen \& Trilling argue that during later evolutionary stages during the planet formation the accretion rate will decrease, allowing for a disk disruption. However, we like to point out that a lower accretion rate also implies a lower mass loss rate for the outflow.

The previous estimates demonstrate again how critical the scenario of protoplanetary outflow formation depends on the circum-planetary accretion rate. More detailed numerical simulations of the circum-planetary accretion disk evolution are essential in order to clarify this point. So far, we have to rely on the simulations presented in the literature which give a high accretion rate.
Although we have shown that it is unlikely that a protoplanetary magnetic field can open up a gap in the circum-planetary accretion disk it is interesting to calculate the co-rotation radius $r_{\mathrm{co}}$ of such a magnetosphere. For a rotational period of the protoplanet of $P_{\mathrm{p}}=10^{\mathrm{h}}$, we find

$r_{\mathrm{co}}=2.25 R_{\mathrm{J}}\left(\frac{M_{\mathrm{p}}}{M_{\mathrm{J}}}\right)^{1 / 3}\left(\frac{P_{\mathrm{p}}}{10^{\mathrm{h}}}\right)^{2 / 3}$,

which is comparable to its radius. The ratio $r_{\mathrm{co}} / R_{\mathrm{J}}$ is about a factor 10 larger than for the protostellar case. Note that breakup rotational period for a $R_{\mathrm{P}}=3 R_{\mathrm{J}}\left(R_{\mathrm{P}}=2 R_{\mathrm{J}}\right)$ protoplanetary core is about $15 \mathrm{~h}(8 \mathrm{~h})$.

As shown above, the circum-planetary disk most probably reaches below the co-rotation radius, implying an angular velocity at the basis of an outflow higher than the one of the central planet - in difference to the case of protostars where the outflow emanates from regions outside the co-rotation radius.

It has been claimed in the literature that a stellar magnetic field sufficiently strong to open up a gap between the star and the disk would be automatically strong enough (e.g. Quillen \& Trilling ) to launch a magnetized wind. However, one has to accept the fact that there is (yet) no numerical proof of such a statement. Stationary state solutions cannot trace the evolution of an outflow (Camenzind 1990; Shu et al. 1994; Fendt et al. 1995) and time-dependent simulations of outflow formation either do not consider a dipolar central field (e.g. Ouyed \& Pudritz 1997) or do not show evidence for a collimated outflow (Miller \& Stone 1997; Goodson et al. 1997; Fendt \& Elstner 2000). The fact that strongly magnetized neutron stars and white dwarfs do not have collimated outflows is another interesting feature in this respect. On the other hand, outflows are observed in active galactic nuclei emanating from the accretion disk around a black hole which does not generate a magnetic field at all.

\subsection{The circum-planetary disk magnetic field}

Circum-stellar accretion disks are believed to carry a substantial magnetic field. Under certain circumstances, this disk field can act as a driver for the observed outflow activity. Further, a disk magnetic field of moderate strength plays the essential role of generating the disk turbulence by the magneto-rotational instability (Balbus \& Hawley 1991) - which enables the disk angular momentum transport and eventually allows for accretion of matter at all. In turn, turbulent motion in a rotating ionized medium may give rise to "mean field" dynamo action ( $\alpha$-effect).

As discussed above (see Sects. 3.2 and 3.3) there exist strong similarities between circum-stellar and circumplanetary accretion disks concerning the degree of ionization and the temperature distribution. In the following we will assume that dynamo action in a circum-planetary disk is indeed feasible. Another possibility to accumulate magnetic flux in the circum-planetary disk is by advection of ambient field (i.e. the magnetic field induced in the surrounding circum-stellar disk) within the accretion stream. 
From Eq. (9) we derive a circum-planetary disk equipartition field strength of

$$
\begin{aligned}
B_{\mathrm{eq}}(r)= & 480 \mathrm{G} \alpha^{-1 / 2}\left(\frac{\dot{M}_{\mathrm{cp}}}{6 \times 10^{-5} M_{\mathrm{J} \mathrm{yr}}-1}\right)^{1 / 2}\left(\frac{M}{M_{\mathrm{J}}}\right)^{1 / 4} \\
& \times\left(\frac{h / r}{0.1}\right)^{-1 / 2}\left(\frac{r}{10 R_{\mathrm{J}}}\right)^{-5 / 4} \cdot
\end{aligned}
$$

This field strength is definitely higher than the surface field strength estimated above for a protoplanetary dynamo, in particular, since we have to consider a turbulence parameter $\alpha<1$. We may therefore conclude that a hypothetical protoplanetary outflow is launched from the disk and not from the planet itself. The actual disk magnetic field strength is certainly below the equipartition field and depends on the detailed properties of the dynamo (see e.g. Rüdiger et al. 1995). In general, the toroidal disk magnetic field strength below the equipartition value and the poloidal field strength one or two orders of magnitude below the toroidal field, with $B_{r} \simeq B_{z}$ depending on the dynamo number. Adding up, this gives a estimate for the circum-planetary accretion disk poloidal magnetic field at $r=10 R_{\mathrm{J}}$ of about $10-50 \mathrm{G}$, if we consider $\alpha \simeq 0.01$.

A disk field strength similar to the equipartition field strength would lead to disk instabilities. The disk magnetic field amplified by the magneto-rotational instability induced turbulence will be saturated by the buoyant escape in vertical direction leading to the formation of a magnetized disk corona (Merloni 2003). Further, disks with high Poynting flux implying a relatively strong poloidal magnetic field are favored by a low viscosity. These results obtained for disks around stellar mass black holes may be tentatively generalized to other parameters regimes. Three-dimensional MHD simulations show that a magnetic torus with initially equipartition field strength will change into a state in which the global field strength is (1/5) of the equipartition field strength (Machida et al. 2000). However, locally, magnetic pressure dominated filaments can be found.

If the differential rotation of the disk ( $\Omega$-effect) completely dominates the magnetic field induction by the dynamo, the magnetic disk flux will be negligible, and the outflow might be launched as a magnetic "plasma gun" (see Contopoulos 1995). If the $\alpha$-effect generates a substantial poloidal field component, we can expect an outflow to be launched similar to the wellknown magneto-centrifugal mechanism (Blandford \& Payne 1982).

\subsection{Magnetization and asymptotic outflow velocity}

The essential parameter determining the asymptotic speed of the MHD outflow, is its magnetization,

$\sigma=\frac{\Phi^{2} \Omega_{\mathrm{F}}^{2}}{c^{3} \dot{M}_{\text {out }}}$

(Michel 1969). Here, $\Omega_{\mathrm{F}}$ is the angular velocity at the foot point radius $r_{\mathrm{F}}$ of a magnetic field line, $\Phi \equiv r_{\mathrm{F}}^{2} B_{\mathrm{F}}$ refers to the magnetic flux from that area and $\dot{M}_{\text {out }}$ to the outflow mass loss rate. For an outflow expanding in spherically radial direction, Michel (1969) derived an analytical relation between the asymptotic flow velocity follows and the magnetization, $v_{\infty} \sim$ $\sigma^{1 / 3}$ (Michel scaling). We emphasize the importance of having a rapid rotation $\left(\Omega_{\mathrm{F}}\right)$ in order to launch a fast outflow. Faster rotators launch faster outflows. It has been shown that the power law interrelation above is maintained also forcollimating outflows, however with a different power index. In fact, the Michel scaling can be applied for a wide range of astrophysical outflows from protostellar outflows with $v_{\text {out }} \simeq 500 \mathrm{~km} \mathrm{~s}^{-1}$ and $\sigma \simeq 10^{-8}$ to the extragalactic case with $v_{\text {out }} \simeq c$ and $\sigma \simeq 10$ (Fendt \& Camenzind 1996).

Applying the Michel scaling for the asymptotic outflow velocity from circum-planetary accretion disks, we obtain

$$
\begin{aligned}
v_{\infty} \simeq & 63 \mathrm{~km} \mathrm{~s}^{-1}\left(\frac{\Phi_{\mathrm{cp}}}{5 \times 10^{22} \mathrm{G} \mathrm{cm}^{2}}\right)^{2 / 3}\left(\frac{P}{4^{\mathrm{d}}}\right)^{-2 / 3} \\
& \times\left(\frac{\dot{M}_{\mathrm{out}}}{10^{-3} \dot{M}_{\mathrm{cp}}}\right)^{-1 / 3}\left(\frac{\dot{M}_{\mathrm{cp}}}{6 \times 10^{-5} M_{\mathrm{J}} \mathrm{yr}}\right)^{-1 / 3}
\end{aligned}
$$

Here, we have considered the magnetic flux $\Phi_{\mathrm{cp}}=5 \times$ $10^{22} \mathrm{G} \mathrm{cm}^{2}$ corresponding to a poloidal magnetic field component of $B_{\mathrm{p}} \simeq 10 \mathrm{G}$ and a Keplerian period at $r=10 R_{\mathrm{J}}$. For the outflow mass flow rate we assume $0.1 \%$ of the disk accretion rate, a typical value derived in MHD outflow formation models. For comparison, the asymptotic speed of an outflow launched from the planetary surface would be $v_{\infty} \simeq 13 \mathrm{~km} \mathrm{~s}^{-1}$ only, with the magnetic flux $\Phi_{\mathrm{P}}=5 \times 10^{20} \mathrm{G} \mathrm{cm}^{2}$ and a rotational period of $10^{\mathrm{h}}$ (present-day Jupiter).

The asymptotic outflow velocity as derived in Eq. (15) actually represents a lower limit. Taking into account a higher disk field strength and a more rapid disk rotation closer to the planet the asymptotic velocity can be larger by a substantial factor.

\subsection{Comparison to simulations of MHD outflow formation}

In general, two approaches have been undertaken to simulate the formation of an MHD outflow. One way is to treat the acceleration and collimation process above the disk and consider the disk "only" as a boundary condition for the mass flow and the magnetic field structure fixed in time. In the case of a monotonous disk magnetic field distribution a collimated outflow evolves out of a slow injection of matter in the disk corona (e.g. Ouyed \& Pudritz 1997; Fendt \& Cemeljic 2002), The other approach includes the evolution of the disk in the simulation and the disk-outflow connection, i.e. the launching mechanism, can be investigated (Hayashi et al. 1996; Miller \& Stone 1997; Kuwabara et al. 2000). However, mainly due to numerical problems with the accretion disk stability, this approach does not yet allow to cover the long-term evolution of the disk-outflow system.

All these simulations are performed considering normalized variables. Having length scales normalized to an "inner disk radius", velocities to the Keplerian velocity at this radius and densities corresponding to a certain, given mass flow rate (Ouyed \& Pudritz 1997), the resulting simulations may be scaled from protostellar outflow to the AGN, supposed that the derived parameters (as Mach number in the disk or outflow and disk temperature) are consistent with the observations. The 

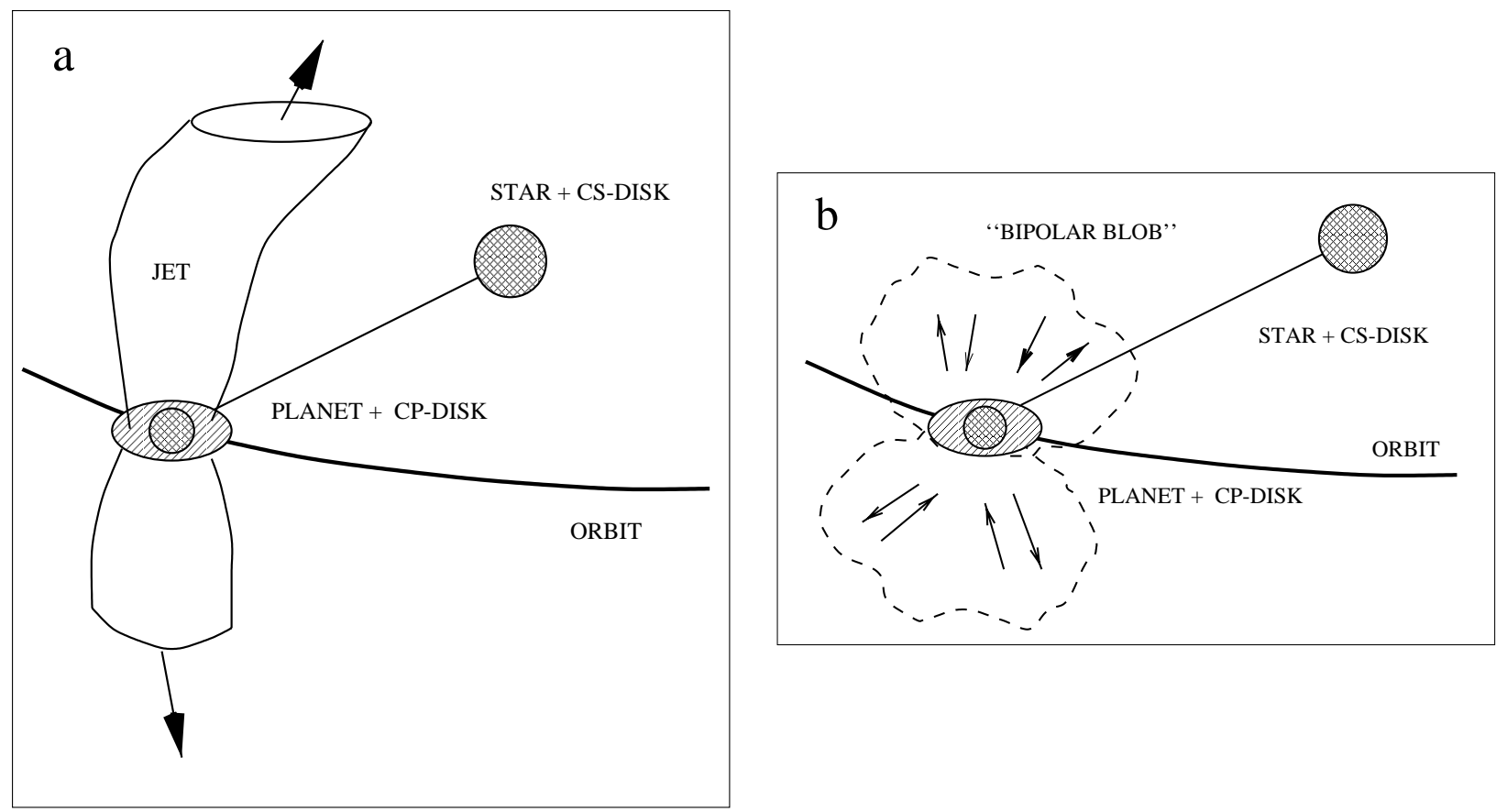

Fig. 1. Model scenario of hypothetical outflows from circum-planetary accretion disks. A protoplanet surrounded by a circum-planetary disk ("CP-disk") is located within the circum-stellar disk ("CS-disk"). Depending on the outflow velocity achieved, two different types of outflow activity are possible - a large scale outflow (a), possibly collimated, or a bipolar blob (b) orbiting with the planet.

question arises whether we can apply recent results of magnetohydrodynamic simulations of outflow formation (e.g. Ouyed \& Pudritz 1997; Fendt \& Elstner 2000; Fendt \& Cemeljic 2002) also for the model scenario of a protoplanet.

Essentially, there are three governing model assumptions involved in the simulations, which are quite well known from the observations. This is the fact that the accretion disk launching the outflow is geometrically thin, implying that the sound speed is small compared to Keplerian speed. Further, the outflow is "cold", i.e. gas pressure can be neglected (low plasma beta). The third assumption concerns the mass load of the outflow. It is clear that we do not know these parameters for the case in the case of planetary outflows due to the (yet existing) lack of observational evidence. As a working hypothesis, we may assume so far that outflow formation works similarly on all scales and that the MHD simulations of protostellar jet formation can indeed be applied for the case of protoplanets. The main results we can extrapolate are then (i) asymptotic outflow velocity several times the Keplerian velocity at the inner disk radius (Ouyed \& Pudritz 1997) and (ii) a rough equipartition between magnetic and kinetic energy in the asymptotic flow. For protoplanetary outflows launched from a disk radius of $10 R_{\mathrm{J}}$ this would imply a asymptotic speed of at least $15 \mathrm{~km} \mathrm{~s}^{-1}$.

\section{Observational appearance of protoplanetary outflows}

Finally, we discuss possible observational features of protoplanetary outflows based on the model scenario developed earlier in this paper. A Jovian mass protoplanetary core surrounded by a circum-planetary accretion disk orbits the central protostar along a Jupiter orbit. Tidal interaction between the protoplanet and the circum-stellar accretion disk has openedup a gap with in the circum-stellar disk. A large scale magnetic field below the equipartition field strength is provided by the circum-planetary accretion disk. The outflow is launched magnetically from the circum-planetary accretion disk.

\subsection{Outflow kinematics and geometry}

A critical measure for the outflow velocity are the escape velocities. The escape velocity of a protoplanetary outflow from the gravitational potential of the protoplanet is

$v_{\mathrm{e}, \mathrm{J}}(r)=59.6 \mathrm{~km} \mathrm{~s}^{-1}\left(\frac{r}{R_{\mathrm{J}}}\right)^{-1 / 2}\left(\frac{M}{M_{\mathrm{J}}}\right)^{1 / 2}$.

This value is similar to the lower limit for the asymptotic flow velocity derived above for the Michel scaling, Eq. (15). In comparison, the escape speed for the protoplanetary outflow from the gravitational potential of the central star at the orbit of Jupiter is

$v_{\mathrm{e}, \star}(r)=18.5 \mathrm{~km} \mathrm{~s}^{-1}\left(\frac{r}{D_{\mathrm{J}}}\right)^{-1 / 2}\left(\frac{M}{M_{\odot}}\right)^{1 / 2}$

Therefore, a protoplanetary outflow propagating faster than the escape velocity from the protoplanet will also be able to leave the gravitational potential of the central star (see Fig. 1a).

For a standard thin circum-stellar accretion disk of aspect ratio $h / r \simeq 0.1$, the scale height at the orbit of Jupiter is $h \simeq$ $0.5 \mathrm{AU} \simeq 210 R_{\mathrm{J}}$. During the early phases of gap formation, the width of that gap will set an upper limit for the radius of the protoplanetary outflow. If the outflow is launched during later 
stages of gap formation, MHD hoop-stresses may collimate the outflow to a radius smaller then the gap size.

During one orbital period a protoplanetary outflow would propagate "length" of the outflow, is

$$
l_{\text {out }, \mathrm{J}}=v_{\infty} P_{\text {orb }, \mathrm{J}}=150 \mathrm{AU}\left(\frac{v_{\infty}}{60 \mathrm{~km} \mathrm{~s}^{-1}}\right)
$$

("length" of the outflow). This is a substantial distance if we consider the size of the outflow origin of only several protoplanetary radii. If the life time of outflow is long enough (i.e. lasting more than several orbits), we expect the overall structure of the outflow to be a hollow tube $e^{2}$ perpendicular to the circum-stellar accretion disk with a diameter of the order of the orbital radius. The protoplanetary jet propagates within a layer along the tube/cone. The thickness of that layer is defined by the radius of the intrinsic outflow and is probably several hundred planetary radii, or about $1 \%$ of the tube diameter. Here we see a major difference to protostellar jets where the mass flow is distributed over the whole width of the global structure.

For an outflow velocity too low in order to leave the planetary gravitational potential, $v_{\mathrm{esc}, \mathrm{J}}>v_{\text {out }}$, the material ejected from the disk will most probably be accumulated in a blob around the planet (see Fig. 1b). As the blob remains bound to the planet, it will follow the orbit around the central star. The actual size of the blob will depend on the detailed balance between ejection of material out of the circum-planetary disk and (spherical?) accretion of this matter back to the protoplanetary system. The maximum size of the blob is the Hill radius. The possible existence of such an extended sphere of hot, shocked gas around the protoplanet is quite interesting from the observational point of view.

The kinetic luminosity $P_{\text {kin }} \simeq \dot{M}_{\text {out }} v_{\text {out }}^{2}$ of a protoplanetary outflow is about

$$
\begin{aligned}
P_{\text {kin }} \simeq & 10^{30} \mathrm{erg} \mathrm{s}^{-1}\left(\frac{\dot{M}_{\text {out }}}{10^{-2} \dot{M}_{\mathrm{cp}}}\right)\left(\frac{\dot{M}_{\mathrm{cp}}}{6 \times 10^{-5} M_{\mathrm{J} \mathrm{yr}}{ }^{-1}}\right) \\
& \times\left(\frac{v_{\text {out }}}{60 \mathrm{~km} \mathrm{~s}^{-1}}\right)^{2} .
\end{aligned}
$$

In comparison, for protostellar outflows with $v_{\text {out }} \simeq 500 \mathrm{~km} \mathrm{~s}^{-1}$ and $\dot{M}_{\text {out }} \simeq 10^{-8} M_{\odot} \mathrm{yr}^{-1}$ this value is four orders of magnitude higher. We therefore conclude that a protoplanetary outflow could be detected only if launched during a time period when no protostellar outflow is present. Otherwise the stellar outflow launched in the inner part of the circum-stellar disk and expanding outwards to about $50 \mathrm{AU}$ will just disrupt the narrow protoplanetary outflow.

An essential property of magnetic winds and outflows is the ability to remove angular momentum very efficiently. This is due to the large lever arm, which is defined by the Alfén radius $r_{\mathrm{A}}$ and typically a factor ten larger then the foot point

\footnotetext{
${ }^{2}$ Depending on the separation of the planet from the central star, the outflow geometry may be characterized better as a hollow cone. Centrifugal imbalance with the central protostar will open up the tube geometry into a cone as the outflow ascends from the ecliptic plane (see Fendt \& Zinnecker 1998; Masciadri \& Raga 2002 for a similar discussion in the context of outflows from binary protostars).
}

radius of the corresponding magnetic field line. The angular momentum carried away per unit mass is

$$
\begin{aligned}
L & =r v_{\phi}-\frac{r B_{\mathrm{p}} B_{\phi}}{4 \pi \rho v_{\mathrm{p}}}=\Omega_{\mathrm{F}} r_{\mathrm{A}}^{2} \\
& =9.5 \times 10^{18} \mathrm{~cm}^{2} \mathrm{~s}^{-1}\left(\frac{M_{\mathrm{p}}}{M_{\mathrm{J}}}\right)^{1 / 2}\left(\frac{r_{\mathrm{F}}}{10 R_{\mathrm{J}}}\right)^{1 / 2}\left(\frac{r_{\mathrm{A}}}{10 r_{\mathrm{F}}}\right)^{2}
\end{aligned}
$$

where $\Omega_{\mathrm{F}}$ is the angular velocity at the foot point radius $r_{\mathrm{F}}$ of the field line. This is a factor $\left(r_{\mathrm{A}} / r_{\mathrm{F}}\right)^{2}$ higher than in the pure hydrodynamic case with $L \simeq \Omega_{\mathrm{F}} r_{\mathrm{F}}^{2}$.

The total angular momentum flux $\dot{J} \equiv \dot{M} L$ carried away by the protoplanetary outflow can be estimated as

$$
\begin{aligned}
\dot{J}_{\text {out }} \simeq & 3 \times 10^{35} \mathrm{~g} \mathrm{~cm}^{2} \mathrm{~s}^{-2}\left(\frac{\dot{M}_{\text {out }}}{10^{-2} \dot{M}_{\mathrm{cp}}}\right)\left(\frac{\dot{M}_{\mathrm{cp}}}{6 \times 10^{-5} M_{\mathrm{J}} \mathrm{yr}^{-1}}\right) \\
& \times\left(\frac{r_{\mathrm{A}}}{10 r_{\mathrm{F}}}\right)^{2}\left(\frac{r_{\mathrm{F}}}{10 R_{\mathrm{J}}}\right)^{1 / 2}\left(\frac{M_{\mathrm{p}}}{M_{\mathrm{J}}}\right)^{1 / 2} .
\end{aligned}
$$

With that, the total angular momentum loss over a orbital period at a Jupiter distance is about $J_{\text {out }}=10^{44} \mathrm{~g} \mathrm{~cm}^{2} \mathrm{~s}^{-2}$.

In comparison, the (rotational) angular momentum of Jupiter is $J_{\mathrm{J}}=6.3 \times 10^{45} \mathrm{~g} \mathrm{~cm}^{2} \mathrm{~s}^{-2}$ (approximated by a homogeneous sphere). Even if the mass carried away by the protoplanetary outflow is small compared to the mass of the central planet, the angular momentum of both components can be of similar order. This leads to the conclusion that a planetary outflow launched for many orbital periods of the protoplanet may well affect the angular momentum evolution of the planetary core and, thus, the time scale for planet formation.

\subsection{Outflow density and observational features}

The time scale of the circum-planetary accretion disk can be roughly estimated to be of the order of

$\tau_{\text {disk }} \simeq M_{\mathrm{J}} / \dot{M}_{\mathrm{cp}} \simeq 2 \times 10^{4} \mathrm{yr} \simeq 1400 P_{\text {orb }, \mathrm{J}}$.

Since the accretion rate decreases with time this value provides only a lower limit. It is clear, that the life time of the disk provides a natural upper limit for the life time of the outflow ${ }^{3}$. The amount of mass deposited by the outflow into the ambient medium during its life time $\tau_{\text {out }}$ is

$$
M_{\mathrm{out}} \simeq \dot{M}_{\mathrm{out}} \tau_{\mathrm{out}} \simeq 10^{-5} M_{\mathrm{J}}\left(\frac{\tau_{\mathrm{out}}}{10^{-3} \tau_{\mathrm{disk}}}\right)\left(\frac{\dot{M}_{\mathrm{out}}}{10^{-2} \dot{M}_{\mathrm{cp}}}\right) .
$$

On the other hand, from the outflow mass accumulated during one orbital period being confined in a $100 R_{\mathrm{J}}$-wide surface layer along the global outflow tube of $10 \mathrm{AU}$ diameter and $150 \mathrm{AU}$ length, we derive mean a particle density within this layer of about $2800 \mathrm{~cm}^{-3}$. This value is surprisingly similar to protostellar outflows (see e.g. Mundt et al. 1990). As the velocities are not too different from protostellar jets, we therefore expect the existence of shock excited optical forbidden lines also in protoplanetary outflow. The observed features would, however,

\footnotetext{
3 Statistical examinations as well as the kinematic evolution of protostellar jets show that the life time of the outflow must be considerably shorter than the disk time scale.
} 
look different. In protostellar outflows we observe a wide shock structure extending over the whole $50 \mathrm{AU}$ width of the outflow. In comparison, in the case protoplanetary outflows one has to consider a bunch of "shocklets" distributed around the hollow tube of the flow. As the kinetic luminosity of the proposed protoplanetary outflows is at least two orders of magnitude lower than for protostellar outflows, we expect the same for luminosity in the shock emission. However, as discussed above, one can suspect that protostellar outflows are launched at times scales earlier than those considered the formation of protoplanets and their hypothetical outflows.

\subsection{Influence on the planet formation process}

Can protoplanetary outflows affect the process of planet formation? Basically, the outflow restores accreted matter back into the ambient medium while efficiently removing angular momentum from the accretion disk.

Considering the number values derived above we come to the conclusion that the mass evolution of the planet cannot be affected by the mass loss of the outflow as it is only about $1 \%$ of the accretion rate.

The impact of the enhanced angular momentum loss due to the magnetic outflow can be twofold. First, the time-scale for the accretion process can be decreased as the disk angular momentum is removed more efficiently compared to purely viscous transport in the disk. The acceleration in the growth rate for the protoplanet is difficult to estimate. A first guess would be that this time-scale is lowered by the same factor of 100 at which the angular momentum loss is increased. Secondly, the angular momentum loss by the disk wind will influence the planet rotational period. If angular momentum is redistributed into the interstellar medium and, thus, not accreted by the planetary core, the final planet can be expected to rotate slower.

\section{Summary}

In this paper we have considered the possibility of launching magnetized outflows from circum-planetary accretion disks. We discuss a model scenario where the protoplanet is accompanied by a circum-planetary accretion disk which is fed from the surrounding circum-stellar accretion disk. This scenario is motivated by recent numerical simulations of planet formation.

For the outflow formation itself we suppose a mechanism similar to other astrophysical outflows (protostellar jets, extragalactic jets) where the flow is magnetically launched as a disk wind and then accelerated and collimated into a narrow beam by Lorentz forces.

We have estimated the magnetic field strength of a fully convective protoplanetary core and find an upper limit for the surface field strength of about $10 \mathrm{G}$. For the surrounding circum-planetary accretion disk we assume a accretion rate of $\dot{M}_{\mathrm{cp}}=6 \times 10^{-5} M_{\mathrm{J}}$ yr for Jupiter-mass protoplanet. The equipartition magnetic field strength in such a disk is of a few $100 \mathrm{G}$ and larger than the protoplanetary magnetic field. The magnetic flux from the circum-planetary accretion disk is estimated to $5 \times 10^{22} \mathrm{G} \mathrm{cm}^{2}$.
We further investigated the circum-planetary accretion disk temperature, its ionization state and magnetic Reynolds number. The circum-planetary disk temperature may reach values up to $2000 \mathrm{~K}$. We find strong indication for a sufficient matterfield coupling underlining the magnetic character of the disk. The latter is an essential condition for outflow launching.

From the estimated accretion rate and magnetic field strength we consider the outflow magnetization for the asymptotic outflow velocity. Applying the Michel scaling for magnetic outflows this velocity is about $63 \mathrm{~km} \mathrm{~s}^{-1}$ and of the order of the escape speed for the protoplanet. However, a modified Michel scaling implied by the collimated structure of the flow may result in even higher velocities.

For reasonable estimates for the outflow mass loss rate of $\dot{M}_{\text {out }}=10^{-2} \dot{M}_{\text {cp }}$ and a velocity of $v_{\text {out }}=60 \mathrm{~km} \mathrm{~s}^{-1}$, the estimated kinetic power of a protoplanetary disk outflow is $P_{\text {kin }} \simeq$ $10^{30} \mathrm{erg} \mathrm{s}^{-1}$. These estimates also imply that during one orbit of the planet a mass of about $10^{-5}$ Jupiter masses can be deposited in the interstellar space by the protoplanetary outflow. In the same time period this outflow would travel about $150 \mathrm{AU}$.

Two outflow scenarios are feasible depending on the outflow velocity. If the outflow cannot escape the protoplanetary gravitational potential, we expect the flow to build up a extended (bipolar) blob of hot gas orbiting with the planet. If the outflow exceeds the escape speed of the protoplanet, a collimated outflow may be formed which (at a planetary orbit of about $5 \mathrm{AU}$ ) can also escape the stellar potential.

The overall outflow geometry built up by the orbiting source of a fast outflow is that of a hollow tube or hollow cone perpendicular to both circum-planetary and circum-stellar disk. For the jet mass loss rate considered we find particle densities in the layer along the tube of the outflow of $2800 \mathrm{~cm}^{-3}$, a value similar to the density in protostellar jets. Shock excited forbidden line emission can therefore be expected.

Energetically protoplanetary outflows cannot survive the interaction with a protostellar jet which is launched from the inner regions of the circum-stellar accretion disk and can, thus, be only present if the stellar outflow has ceased to exist.

The efficient angular momentum loss by the magnetized disk wind may affect the accretion time scale and, thus, the time scale for planet formation. The angular momentum loss by the disk wind or outflow per one orbital period is about the rotational angular momentum of today's Jupiter.

In summary, our model estimates strongly rely on the accretion rate of the circum-planetary accretion disk. We apply $\dot{M}_{\text {cp }}=6 \times 10^{-5} M_{\mathrm{J}}$ yr, a value which is supported by many independent numerical simulations of planet formation in accretion disks. Further model constraints can be expected from future, high resolution numerical simulations of the circum-planetary accretion disk clarifying the detailed circum-planetary disk structure close to the protoplanet.

Acknowledgements. The author thanks Pavel Kroupa for directing his interest to the question of protoplanetary outflows. The present work has benefited from discussions with Detlef Elstner, Willy Kley, Matthias Steffen and, in particular, Gennaro D'Angelo. Hans Zinnecker and Joachim Wambsganss are acknowledged for their continuous support. The paper also improved from insightful 
comments by the referee Rachid Ouyed. This work has been financed by the research grant HSPN 24-04/302;2000 of the State of Brandenburg.

\section{Appendix A: The standard disk parameters}

Here we apply the Shakura \& Sunyaev (1973) accretion disk parametrisation of a thin $\alpha$-viscosity disk to the case of circumplanetary disks. We show the temperature and density profile and for comparison the same also for the circum-stellar disk. In our case radiation pressure can be neglected. Free-free absorption dominates electron scattering. The sound speed is given by $c_{\mathrm{s}}=\left(k_{\mathrm{B}} T / m_{\text {prot }}\right)$. The particle density is

$$
\begin{aligned}
n(r)= & 1.6 \times 10^{14} \mathrm{~cm}^{-3} \alpha^{-7 / 10}\left(\frac{\dot{M}}{6 \times 10^{-5} M_{\mathrm{J}} \mathrm{yr}^{-1}}\right)^{11 / 20} \\
& \times\left(\frac{M}{M_{\mathrm{J}}}\right)^{5 / 8}\left(\frac{r}{15 R_{\mathrm{J}}}\right)^{-15 / 8} \\
= & 2.8 \times 10^{14} \mathrm{~cm}^{-3} \alpha^{-7 / 10}\left(\frac{\dot{M}}{1.2 \times 10^{-7} M_{\odot} \mathrm{yr}^{-1}}\right)^{11 / 20} \\
& \times\left(\frac{M}{M_{\odot}}\right)^{5 / 8}\left(\frac{r}{15 R_{\odot}}\right)^{-15 / 8}
\end{aligned}
$$

while the disk temperature is

$$
\begin{aligned}
T(r)= & 2800 \mathrm{~K} \alpha^{-1 / 5}\left(\frac{\dot{M}}{6 \times 10^{-5} M_{\mathrm{J}} \mathrm{yr}^{-1}}\right)^{3 / 10} \\
& \times\left(\frac{M}{M_{\mathrm{J}}}\right)^{1 / 4}\left(\frac{r}{15 R_{\mathrm{J}}}\right)^{-3 / 4} \\
= & 3700 \mathrm{~K} \alpha^{-1 / 5}\left(\frac{\dot{M}}{1.2 \times 10^{-7} M_{\odot} \mathrm{yr}^{-1}}\right)^{3 / 10} \\
& \times\left(\frac{M}{M_{\odot}}\right)^{1 / 4}\left(\frac{r}{15 R_{\odot}}\right)^{-3 / 4} .
\end{aligned}
$$

The temperature derived here is the mid-plane disk temperature and is in agreement with the simple estimate of the surface temperature in Eq. (3), but also the more sophisticated disk model probing the vertical circum-stellar disk structure (D'Alessio et al. 1998). We note, however, that in spite of the general success of the Shakura \& Sunyaev $\alpha$-parametrization of turbulence, in particular for hot disks around compact objects, it is known that the this model does not fit very well all of the observed disks of T Tauri stars (i.e. cool disks), in which a $T(r) \sim r^{-1 / 2}$ temperature profile indicated (Beckwith et al. 1990).

Finally, we supply the expression for the disk-half thickness from Shakura \& Sunyaev for the case of a circum-planetary disk,

$$
\begin{aligned}
h(r)= & 6.5 \times 10^{6} \mathrm{~cm} \alpha^{-1 / 10}\left(\frac{\dot{M}}{6 \times 10^{-5} M_{\mathrm{J}} \mathrm{yr}^{-1}}\right)^{3 / 20} \\
& \times\left(\frac{M}{M_{\mathrm{J}}}\right)^{9 / 10}\left(\frac{r}{15 R_{\mathrm{J}}}\right)^{9 / 8} .
\end{aligned}
$$

Compared to the circum-stellar accretion disk, the circumplanetary is expected to somewhat thinner.

\section{References}

Artymowicz, P., \& Lubow, S. H. 1994, ApJ, 421, 651

Artymowicz, P., \& Lubow, S. H. 1996, ApJ, 476, L77

Balbus, S. A., \& Hawley, J. F. 1991, ApJ, 376, 214

Bate, M. R., Lubow, S. H., Ogilvie, G. I., \& Miller, K. A. 2003, MNRAS, 341, 213

Beckwith, S. V. W., Sargent, A. I., Chini, R. S., \& Guesten, R. 1990, AJ, 99, 924

Blackman, E. 2003, Recent Developments in Magnetic Dynamo Theory, in Turbulence and Magnetic Fields in Astrophysics, ed. E. Falgarone, \& T. Passot, Lect. Notes in Phys., 614, 432 [astro-ph/0205002v2]

Blandford, R. D., \& Payne, D. G. 1982, MNRAS, 199, 883

Bouvier, J. 1990, AJ, 99, 946

Bryden, G., Chen, X., Lin, D. N. C., Nelson, R. P., \& Papaloizou, J. C. B. 1999, ApJ, 514, 344

Burrows, A., Hubbard, W. B., Lunine, J. I., \& Liebert, J. 2001, RvMP, 73, 719

Camenzind, M. 1990, Rev. Mod. Astron. 3, 234

Camenzind, M. 1997, Energetics, Collimation and Propagation of Galactic Protostellar Outflows. Views and Perspectives, in Herbig-Haro Flows and the Birth of Stars, ed. B. Reipurth, \& C. Bertout, IAU, 182 (Kluwer), 241

Canup, R. M., \& Ward, W. R. 2002, AJ, 124, 3404

Ciecielag, P., Plewa, T., \& Różyczka, M. 2000 High-resolution Simulations and Visualization of Protoplanetary Disks, in Birth and Evolution of Binary Stars, ed. B. Reipurth, \& H. Zinnecker, IAU, 200, poster proceedings, 199

Connerney, J. E. P. 1993, Journal of Geophys. Res., 98, 18-659

Contopoulos, J. 1995, ApJ, 450, 616

D’Angelo, G., Henning, T., \& Kley, W. 2002, A\&A, 385, 647

D'Angelo, G. 2003, private communication

D’Angelo, G., Kley, W., \& Henning, T. 2003, ApJ, 586, 540

D’Angelo, G., Henning, T., \& Kley, W. 2003, ApJ, 599, in press [astro-ph/0309236]

D’Alessio, P., Cantó, J., Calvet, N., \& Lizano, S. 1998, ApJ, 500, 411

Dullemond, C. P., van Zadelhoff, G. J., \& Natta, A. 2002, A\&A, 389, 464

Fendt, Ch., Camenzind, M., \& Appl, S. 1995, A\&A, 300, 791

Fendt, Ch., \& Camenzind, M. 1996, A\&A, 313, 591

Fendt, Ch., \& Zinnecker, H. 1998, A\&A, 334, 750

Fendt, Ch., \& Elstner, D. 2000, A\&A, 363, 208

Fendt, Ch., \& Cemeljic, M. 2002, A\&A, 395, 1043

Fromang, S., Terquem, C., \& Balbus, S. 2002, MNRAS, 329, 18

Gammie, C. F. 1996, ApJ, 457, 355

Ghosh, P. 1995, MNRAS, 272, 763

Goodson, A. P., Winglee, R. M., \& Böhm, K.-H. 1997, ApJ, 489, 199

Grote, E., Busse, F. H., \& Tilgner, A. 1999, Phys. Rev. E, 60, R5025

Hayashi, M. R., Shibata, K., \& Matsumoto, R. 1996, ApJ, 468, L37

Hubeny, I. 1990, ApJ, 351, 632

Kirk, R. L., \& Stevenson, D. J. 1987, ApJ, 316, 836

Kley, W. 1999, MNRAS, 303, 696

Kley, W., D’Angelo, G., \& Henning, T. 2001, ApJ, 547, 457

Königl, A. 1991, ApJ, 370, 39

Kuwabara, T., Shibata, K., Kudoh, T., \& Matsumoto, R. 2000, PASJ, 52, 1109

Kudoh, T., Matsumoto, R., \& Shibata, K. 1998, ApJ, 508, 186

Küker, M., \& Rüdiger, G. 1997, A\&A, 328, 253

Küker, M., \& Rüdiger, G. 1999, A\&A, 346, 922

Lin, D. N. C., \& Papaloizou, J. C. B. 1986, ApJ, 307, 395

Lin, D. N. C., \& Papaloizou, J. C. B. 1993, On the Tidal Interaction between Protostellar Disks and Companions, in Protostars and Planets III, ed. E. H. Levy, \& J. I. Lunine (Tucson: Univ. of Arizona Press), 749 
Lubow, S. H., Seibert, M., \& Artymowicz, P. 1999, ApJ, 526, 1001 Machida, M., Hayashi, M. R., \& Matsumoto, R. 2000, ApJ, L67 Malbet, F., Lachaume, R., \& Monin, J.-L. 2001, A\&A, 379, 515 Masciadri, E., \& Raga, A. C. 2002, ApJ, 568, 733

Merloni, A. 2003, MNRAS, 341, 1051

Meyer, F., \& Meyer-Hofmeister, E. 1994, A\&A, 288, 174

Michel, F. C. 1969, ApJ, 158, 727

Miller, K. A., \& Stone, J. M. 1997, ApJ, 489, 890

Moss, D., \& Brandenburg, A. 1995, Geophys. Astrophys. Fluid Dynam., 80, 229

Mundt, R., Bührke, T., Solf, J., Ray, T., \& Raga, A. C. 1990, A\&A, 232, 37

Nelson, R. P., Papaloizou, J. C. B., Masset, F., \& Kley, W. 2000, MNRAS, 318, 18

Nelson, A. F., \& Benz, W. 2003, ApJ, 589, 578

Ouyed, R., \& Pudritz, R. E. 1997, ApJ, 482, 712

Ouyed, R., Clarke, D., \& Pudritz, R. E. 2003, ApJ, 582, 292

Pizzolato, N., Maggio, A., Micela, G., Sciortino, S., \& Ventura, P. 2003, A\&A, 397, 147
Pringle, J. 1981, ARA\&A, 19, 137

Pudritz, R. E., \& Norman, C. A. 1986, ApJ, 301, 571

Quilling, A. C., \& Trilling, D. E. 1998, ApJ, 508, 707

Rüdiger, G., Elstner, D., \& Stepinski, T. F. 1995, A\&A, 298, 934

Shakura, N. I., \& Sunyaev, R. A. 1973, A\&A, 24, 337

Shu, F. H., Najita, J., Wilkin, F., Ruden, S. P., \& Lizano, S. 1994, ApJ, 429, 781

Starchenko, S. V., \& Jones, C. A. 2002, Icarus, 157, 426

Tanigawa, T., \& Watanabe, S.-I. 2002, ApJ, 580, 506

Vishniac, E. T., Lazarian, A., \& Cho, J. 2003, Problems and Progress in Astrophysical Dynamos, in Turbulence and Magnetic Fields in Astrophysics, ed. E. Falgarone, \& T. Passot, Lect. Notes in Phys., 614, 376 [astro-ph/0205557v1]

Wuchterl, G., Guillot, T., \& Lissauer, J. J. 2000, Giant planet formation, in Protostars and Planets IV, ed. V. Mannings et al. (Tuscon: Univ. of Arizona Press), 1081 